\title{
Interannual and spatial variation in the parasite communities of Pacific sierra Scomberomorus sierra (Jordan et Starks) on Mexico's Pacific coast
}

\author{
Nataly G. Santos-Bustos ${ }^{1}$, Juan Violante-González ${ }^{1,2^{*}}$, Scott Monks ${ }^{3}$, Princessa J. Villalba-Vásquez ${ }^{1}$, Shirley S. \\ Salas Villalobos ${ }^{2}$, Monserrat S. Acosta-Hernández ${ }^{2}$ and Aldo Diaz Gallegos ${ }^{2}$ \\ ${ }^{1}$ Centro de Ciencias de Desarrollo Regional, Universidad Autónoma de Guerrero, Acapulco, México; \\ ${ }^{2}$ Facultad de Ecología Marina, Universidad Autónoma de Guerrero, Acapulco, Guerrero, México; \\ ${ }^{3}$ Centro de Investigaciones Biológicas, Universidad Autónoma del Estado de Hidalgo, Pachuca, Hidalgo, México
}

\begin{abstract}
The parasite communities of predatory fish can be species rich and diverse, making them effective models for studying the factors influencing temporal and spatial variation in these communities. Over a ten-year period an initial study was done on the metazoan parasite communities of Scomberomorus sierra (Jordan et Starks) from four locations on the south-central Pacific coast of Mexico. Twenty-four metazoan parasite taxa were identified from 674 S. sierra specimens: three species of Monogenea, eight Digenea, one Cestoda, one Acanthocephala, four Nematoda, five Copepoda, and two Isopoda. The parasite communities were characterised by high ectoparasite species richness, with monogeneans and some didymozoid species being numerically dominant. Community structure and species composition varied between locations, seasons and sampling years. Similarity between the component parasite communities was generally low, despite the occurrence of a distinctive set of host-specialist parasites. Interannual or local variations in some biotic and abiotic environmental factors are possible causes of the observed variations in the structure and species composition of the parasite community of S. sierra. Ecological factors were therefore considered to have more influence than phylogenetic aspects (host phylogeny) on parasite community structure.
\end{abstract}

Keywords: Marine parasites, predatory fish, tropics, south-central Pacific, Mexico.

Marine metazoan parasite communities consist largely of ectoparasites (monogeneans, copepods and isopods) and endoparasites (digeneans, cestodes, nematodes and acanthocephalans) (Poulin and Rohde 1997, Rohde 2005). In each of these parasite groups species richness and diversity vary substantially between different host species (Poulin and Rohde 1997, Rohde 2005). For example, ectoparasite populations may be more abundant in fish with schooling behaviour than in solitary species because the probability of a transmission stage (e.g. eggs, larvae) contacting a host increases with greater host density (Sasal et al. 1997, Luque et al. 2004, Palacios-Fuentes et al. 2015). This has been reported in several tropical monogenean species such as Pseudomazocraes selene Hargis, 1957, Parancylodiscoides chaetodipteri Caballero et Bravo-Hollis, 1961, Protomicrocotyle manteri Bravo-Hollis, 1966 and Neomicrocotyle pacifica Meserve, 1938 (Gallegos-Navarro et al. 2018, Villalba-Vásquez et al. 2018, Violante-González et al. 2020), as well as several species of copepods of the genus Caligus Müller, 1785 (Palacios-Fuentes et al. 2015).
Understanding the factors that influence parasite community structure and organisation in marine fish populations is a primary focus in parasite ecology. Parasite communities can exhibit temporal and spatial changes in their species composition and structure due to seasonal and local variations in several biotic and abiotic environmental factors (Garcias et al. 2001, Henríquez and González 2012, Villalba-Vásquez et al. 2018). In the tropics, structural changes in these communities have been linked principally to host traits such as age, body size, feeding behaviour, host density and vagility. These traits can promote exposure to new parasite species, increase their colonisation rates, and are associated with parasite communities of greater diversity and species richness (Luque et al. 2004, Villalba-Vásquez et al. 2018).

Several studies indicate that trophic level and diet determine the prey range of a fish predator. Many parasite species, particularly endoparasites, have complex life cycles involving transmission through a trophic web of intermediate, paratenic and definitive hosts (Luque and Poulin 2008,

Address for correspondence: J. Violante-González, Facultad de Ecología Marina. Gran Vía Tropical Núm. 20, Fracc. Las Playas, C. P. 39390. Universidad Autónoma de Guerrero, Acapulco, Guerrero, México; email: viojuang@yahoo.com.mx 


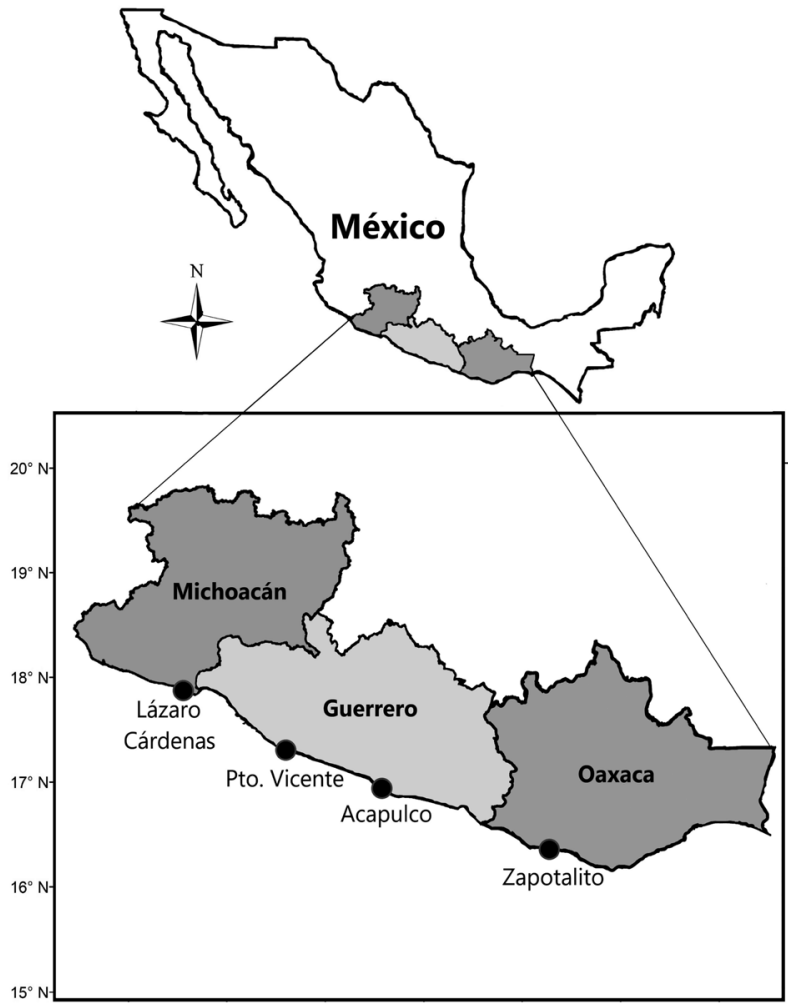

Fig. 1. Geographic locations of the sample collections of Scomberomorus sierra (Jordan et Starks) alongside the Mexico's Pacific coast.

Timi et al. 2011). Over evolutionary time host species at higher trophic levels (i.e. carnivorous and piscivorous fish) would therefore be exposed through diet to greater numbers of infective endohelminth larvae from a broader range of parasite taxa than would those at lower levels (Luque and Poulin 2008, Timi et al. 2011).

The Pacific sierra, Scomberomorus sierra Jordan et Starks (Scombridae), is a pelagic-neritic fish that forms schools of similarly-aged individuals. It is widely distributed in the eastern Pacific from southern California, USA, to the Galapagos Islands and Paite, Peru. Adults feed mainly on small fish such as anchovies and sardines (Collette and Nauen 1983, Moreno-Sánchez et al. 2011), but it is considered an opportunistic predator (i.e. one able to feed on prey that is unusually abundant in its environment). Along the Pacific coast of Mexico S. sierra is a major fishery (Aguirre-Villaseñor et al. 2006, Moreno-Sánchez et al. 2011). Some parasites of $S$. sierra has been reported for at least two locations for this region (Pérez-Ponce de León et al. 1999, Bárcenas 2019), but spatial and temporal variation over long time periods (interannual scale) has not yet been studied in this species.

The present study addressed two main objectives: (1) to characterise the metazoan parasite communities of $S$. sier$r a$, and (2) to evaluate spatial and temporal variability in its parasite communities.

\section{MATERIALS AND METHODS}

A total of 674 specimens of Scomberomorus sierra were obtained from commercial fishermen as twelve samples (March
2011; March and August 2012; November 2013; February and December 2017; January, April and December 2018; January and May 2019; February 2020) over a 10-year period from four locations along the Pacific coast of Mexico: Zapotalito, Oaxaca $(15.9686 \mathrm{~N}, 97.5380 \mathrm{~W}, n=170)$; Acapulco Bay $(16.8524 \mathrm{~N}$, 99.8823W, $n=391)$, Puerto Vicente $(17.2677 \mathrm{~N}, 101.0519 \mathrm{~W}, n$ = 64) in Guerrero; and Lázaro Cárdenas, Michoacán (17.9161N, 102.2033W, $n=49$ ) (Fig. 1). Records of sea surface temperature per sampling date were obtained from other studies carried out in the same locations but not yet published. Multivariate El Niño index values (MEI) for each sampling date were obtained from the National Oceanic and Atmospheric Administration (NOAA: https://www.esrl.noaa.gov/psd/enso/mei/table.html). Fish sex, total length (fish body size) and total weight for each individual were measured at the time of collection.

Fish from each sampling location were pooled into three size-classes: immature $(<35 \mathrm{~cm})$, pre-adults $(36-40 \mathrm{~cm})$ and adults $(>40 \mathrm{~cm}$ ) (Aguirre-Villaseñor et al. 2006). The coefficient of variation $(\mathrm{CV})$, i.e. the quotient between the standard deviation and the arithmetic mean, was used to assess the variability in the total length of fish populations in each location or sampling year. Possible differences in the fish body size between sexes, locations and sampling years were determined using a one-way ANOVA.

The Fulton's condition factor (CF) was calculated using the equation: $\mathrm{CF}=\mathrm{W}_{\mathrm{t}} / \mathrm{L}_{\mathrm{t}}^{3}$, where $\mathrm{W}_{\mathrm{t}}$ is total weight, and $\mathrm{L}_{\mathrm{t}}=$ total length. A complete necropsy was done of all specimens and all parasites collected from the internal and external organs. Digestive tract contents were examined to identify prey items consumed by this species at each location. Dietary items were identified to the family level when possible. Prey item analysis was done using the frequency of occurrence method (Lima-Junior and Goitein 2001). Parasites were identified to the lowest possible taxonomic level; vouchers of the most abundant and best preserved specimens were deposited in the in the Colección de Morfología Animal (COMA 00098 to 00110), Centro de Investigaciones Biológicas, Universidad Autónoma del Estado de Hidalgo, México.

Infection levels for each parasite species were described using prevalence (percent of fish infected with a particular parasite species); mean abundance (mean number of individual parasites of a particular species per examined fish), expressed as the mean \pm standard deviation (SD); and intensity (number of a particular parasite species per infected fish), expressed as range (minimum-maximum) (Bush et al. 1997). The total prevalence (i.e. the percentage of infected fish with at least one of the parasite species) and the total mean intensity (mean total number of parasites in each sample) were used to determine possible relationships between the fish total length and these infection parameters within and between locations or sampling years.

Possible differences in infection levels between sampling years and/or locations were identified using $G$-tests (Sokal and Rohlf 1998) for prevalence and a General Linear Model (GLM) for abundance. The normality of data was determined with the Kolmogorov-Smirnov test with the use of Lilliefors' approach (Sokal and Rohlf 1998). When important deviations from normality were found, data were $\log$-transformed $(\ln [X+1])$. The host range concept (Rohde 2005) was used to classify the parasite species as specialists or generalists. In the present study, host-specialist species were those previously reported from scombrid fish 


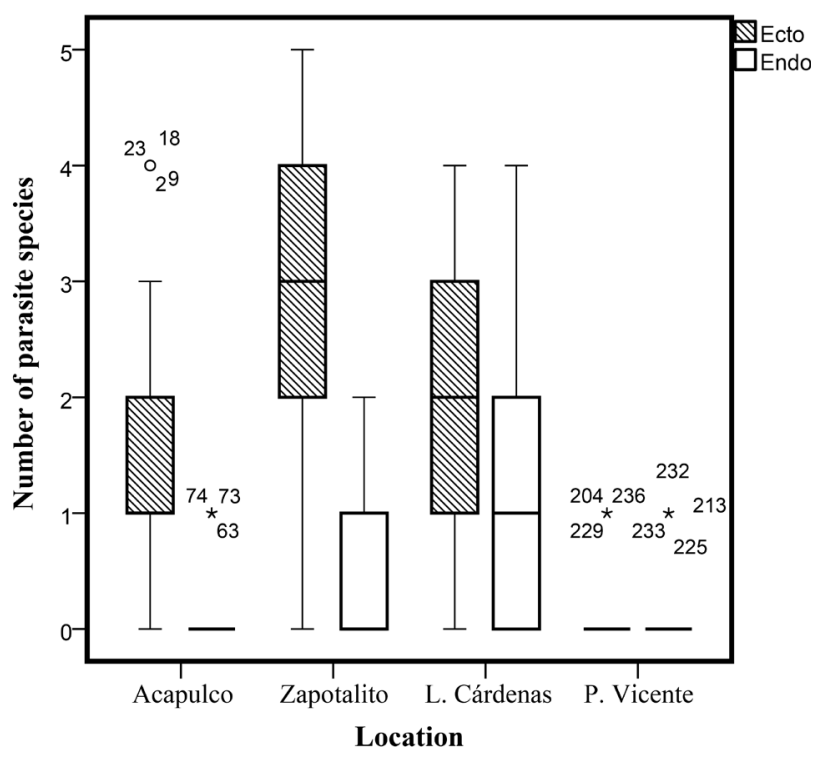

Fig. 2. Box plot of species number of ectoparasites (monogenean, didymozoid and crustacean), and endoparasites (larvae and adults) in Scomberomorus sierra (Jordan et Starks) from the Pacific coast of Mexico. The bottom and top of the boxes represent the lower and upper quartiles respectively; the median is shown as a line through the centre of the boxes; outliers are indicated with circles for ectoparasites and asterisks for endoparasites.

(Scombridae), and generalists were those previously reported from species of fish of different families (Aguirre-Macedo et al. 2007). The infracommunity index (ICI) (Zander 2004), which describes the frequency of double and multiple infections by a single species of parasite in a distinct host (affinity level of a parasite species; i.e. parasite species with great tendency to join the infracommunity), was also calculated. Spearman's correlation coefficient $\left(r_{s}\right)$ was used to determine possible relationships between host total length and the infection or infracommunity parameters.

Analyses were done at the levels of component community (i.e. total parasite species in all fish collected at a location) and infracommunity (i.e. total parasite species in each individual fish). Component community parameters included total species richness; total number of individuals of each parasite species; the Shannon-Wiener Index $(\mathrm{H})$ as a measure of diversity; species evenness (equitability); and the Berger-Parker Index (BPI) as a measure of numerical dominance. The qualitative Sorensen's index and quantitative percentage of similarity (PS) index were used to evaluate similarity and difference in parasite community species composition between years and/or locations. Differences between component community parameters were identified with Student $t$ and $\chi^{2}$ tests.

Infracommunities were described in terms of mean number of parasite species per host, mean number of individuals of each species, and the mean Brillouin Diversity Index ( $\left.\mathrm{H}^{\prime}\right)$ value per host. The multivariate general linear model (GLM) was used to identify possible differences in infracommunity parameters (dependent variables) between locations and/or sampling years (predictor variables); fish body size (total length) was used as a covariate to control for the influence of host body size. The significance of all statistical analyses was established at $p<0.05$, unless stated otherwise. A multivariate analyses (Principal Component Analy- sis, PCA) was applied to identify biotic and abiotic factors that influenced parasite infracommunity species richness and diversity. To minimise the effect of host body size only fish $>35 \mathrm{~cm}$ (i.e. pre-adults and adults) were included in this analyses; so two samples of Acapulco 2018 (January, $\mathrm{n}=84$; and December, $\mathrm{n}=$ 77) and the Puerto Vicente 2018 (April, $n=64$ ) were excluded.

The predictor variables used were Fulton's condition factor (CF); host diet diversity (calculated as the diversity of items consumed by host populations in each sampling location, through the use of the Shannon-Wiener index at the family level); number of didymozoid species; surface temperature; climate anomaly values (MEI); climatic season: (dry season, precipitation $<70 \mathrm{~mm}$, and rainy season, precipitation $\approx 950 \mathrm{~mm}$ ) (Violante-González et al. 2020); sampling year and collection location. The Kaiser-Meyer-Olkin (KMO) test of sampling adequacy for each variable in the model, as well as the Bartlett's test, which evaluates the possibility that there is redundancy among variables, were applied. The variance maximising rotation method was used to produce the two ordination axes. Discriminant function analyses (DFA) based on Mahalanobis distances were used to identify possible differences in parasite community structure between locations (to minimise temporal variation as a source of variability, only one sample from Acapulco 2018 $8_{\mathrm{Jan}}$ and one from Zapotalito 2017 $7_{\mathrm{Feb}}$ were included in this analysis). Only parasite species with a prevalence $>10 \%$ in at least one of the locations (a component species; sensu Bush et al. 1990) were included.

\section{RESULTS}

\section{Species composition}

Twenty-four taxa of metazoan parasites (17 helminths and seven crustaceans) were identified from a total sample of 10,573 individual parasites recovered from 674 Scomberomorus sierra specimens collected from four locations along the south-central Pacific coast of Mexico. Three of these species were Monogenea (adults), eight were Digenea (seven adults and one metacercaria), one was Cestoda (larvae), one was Acanthocephala (adult), four were Nematoda (three adults and one larva), five were Copepoda, and two were Isopoda (Table 1). Species richness was highest among the digeneans, representing 34\% of total recovered species, followed by the copepods (29\%). According to infection site, 13 parasite species were classified as ectoparasites and 11 as endoparasites. Ectoparasite species numbers (Fig. 2) varied widely from $0.05 \pm 0.21$ in hosts from Puerto Vicente to $3.06 \pm 1.12$ in those from Zapotalito (ANOVA $\left.\mathrm{F}_{3,244}=102.69, \mathrm{p}<0.01\right)$.

Of the total parasite species sample, ten were classified as host-specialists (three monogeneans, five digeneans and two copepods; Table 1). Nine parasite species (Mexicotyle mexicana [Meserve, 1938], Thoracocotyle crocea [MacCallum, 1913], Didymocilindrus sp., Didymocystis sp., Lecithochirium microstomum [Chandler, 1935], Prosorhynchoides cybii [Park, 1939], Anisakis sp., Hysterothylacium sp. and Caligus omissus [Cressey et Cressey, 1980]) were widely distributed and occurred in the S. sierra parasite communities at all or most of the sampled locations (Table 1). 
Table 1. Parasite infection parameters for Scomberomorus sierra from Mexican Pacific coasts (Zapotalito, Oaxaca; Acapulco and

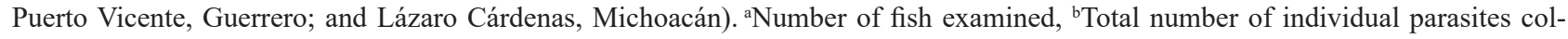
lected, ${ }^{\mathrm{C}} \mathrm{Number}$ of parasites per fish (mean $\pm \mathrm{SD}$ ), ${ }^{\mathrm{d}}$ Minimum to maximum number of parasites present, ${ }^{\mathrm{e}}$ Infracommunity index, ${ }^{\mathrm{f}} \mathrm{New}$ geographical record. Subscripts to the right of the sampling year indicate the sampling month: $\mathrm{J}=$ January, $\mathrm{F}=$ February, Ma $=$ March, Ap = April, $\mathrm{M}=$ May, $\mathrm{A}=$ August, $\mathrm{N}=$ November, $\mathrm{D}=$ December.

\begin{tabular}{|c|c|c|c|c|c|c|c|c|c|c|}
\hline \multirow{2}{*}{$\begin{array}{l}\text { Parasite species } \\
\text { Digenea }\end{array}$} & \multirow[t]{2}{*}{$\begin{array}{l}\text { Parasite } \\
\text { classification }\end{array}$} & \multicolumn{2}{|c|}{ Site on hostLocation } & \multirow[t]{2}{*}{ Year } & \multirow[t]{2}{*}{$\mathrm{n}^{\mathrm{a}}$} & \multirow[t]{2}{*}{$\begin{array}{l}\text { Prevalence } \\
\quad(\%)\end{array}$} & \multirow[t]{2}{*}{ Total $^{\mathrm{b}}$} & \multirow[t]{2}{*}{ Abundance $^{c}$} & \multirow[t]{2}{*}{$\begin{array}{l}\text { Range of } \\
\text { intensity }\end{array}$} & \multirow[t]{2}{*}{$\mathrm{ICI}^{\mathrm{e}}$} \\
\hline & & & & & & & & & & \\
\hline \multirow{7}{*}{$\begin{array}{l}\text { Anacetabulum sp. } \\
\text { (larvae) }\end{array}$} & \multirow[t]{7}{*}{ Specialist } & \multirow[t]{7}{*}{ Gills } & Acapulco & $2012_{\mathrm{A}}$ & 19 & 1 & 2 & 0.03 & $1-2$ & 0.004 \\
\hline & & & Acapulco & $2018_{J}$ & 84 & 1 & 11 & 0.13 & $1-11$ & 0.007 \\
\hline & & & Acapulco & $2019_{\mathrm{M}}$ & 59 & 15 & 19 & $0.32 \pm 1.27$ & $1-4$ & 0.062 \\
\hline & & & Zapotalito & $2017_{\mathrm{F}}^{\mathrm{M}}$ & 48 & 19 & 22 & $0.46 \pm 2.35$ & $1-7$ & 0.054 \\
\hline & & & Zapotalito & $2017_{D}$ & 28 & 7 & 4 & $0.14 \pm 1.41$ & $1-3$ & 0.059 \\
\hline & & & Zapotalito & $2019_{J}$ & 40 & 48 & 59 & $1.48 \pm 3.23$ & $1-14$ & 0.123 \\
\hline & & & Zapotalito & $2020_{\mathrm{F}}$ & 54 & $72 *$ & 564 & $10.44 \pm 22 *$ & $1-109$ & 0.122 \\
\hline Didymocilindrus sp. & Specialist & Gills & Acapulco & $2011_{\mathrm{Ma}}$ & 76 & 67 & 1025 & $13.49 \pm 14.61$ & $5-101$ & 0.216 \\
\hline & & & Acapulco & $2012_{\mathrm{Ma}}$ & 19 & 53 & 87 & $4.58 \pm 4.72$ & $2-15$ & 0.118 \\
\hline & & & Acapulco & $2012_{\mathrm{A}}$ & 76 & 62 & 360 & $4.74 \pm 7.78$ & $1-43$ & 0.199 \\
\hline & & & Acapulco & $2018_{J}$ & 84 & 29 & 100 & $1.19 \pm 4.37$ & $1-17$ & 0.172 \\
\hline & & & Acapulco & $2018_{D}$ & 77 & 3 & 2 & 0.03 & $1-1$ & 0.045 \\
\hline & & & Acapulco & $2019_{\mathrm{M}}$ & 59 & 56 & 154 & $2.61 \pm 4.65$ & $1-22$ & 0.226 \\
\hline & & & Zapotalito & $2017_{\mathrm{F}}$ & 48 & 17 & 52 & $1.08 \pm 4.50$ & $1-13$ & 0.048 \\
\hline & & & Zapotalito & $2017_{D}$ & 28 & 21 & 25 & $0.89 \pm 3.37$ & $2-9$ & 0.177 \\
\hline & & & Zapotalito & $2019_{\mathrm{J}}$ & 40 & 68 & 180 & $4.50 \pm 7.65$ & $1-27$ & 0.175 \\
\hline & & & Zapotalito & $2020_{\mathrm{F}}$ & 54 & $80^{*}$ & 597 & $11.06 \pm 13.7^{*}$ & $1-79$ & 0.135 \\
\hline & & & L. Cárdenas & $2013_{N}$ & 49 & 65 & 258 & $5.27 \pm 6.05$ & $1-32$ & 0.19 \\
\hline Didymocystis sp. & Specialist & Intestine, & Acapulco & $2011_{\mathrm{Ma}}$ & 76 & $67 *$ & 591 & $7.78 \pm 7.90 *$ & $1-48$ & 0.216 \\
\hline & & Stomach, & Acapulco & 2012 & 19 & $74 *$ & 143 & $7.53 \pm 12.44 *$ & $1-47$ & 0.165 \\
\hline & & & Acapulco & 2012 & 76 & 8 & 30 & $0.39 \pm 3.79$ & $2-12$ & 0.025 \\
\hline & & & Acapulco & $2019_{M}$ & 59 & 17 & 49 & $0.83 \pm 4.51$ & $1-15$ & 0.069 \\
\hline & & & Zapotalito & $2019_{\mathrm{J}}^{1 \mathrm{I}}$ & 40 & 13 & 24 & $0.60 \pm 4.82$ & $1-11$ & 0.032 \\
\hline & & & Zapotalito & $2020_{\mathrm{F}}$ & 54 & 54 & 196 & $3.63 \pm 5.93$ & $1-31$ & 0.091 \\
\hline & & & L. Cárdenas & $2013_{N}$ & 49 & 49 & 189 & $3.86 \pm 5.53$ & $2-21$ & 0.14 \\
\hline Didymozoon sp. & Specialist & Intestine & Zapotalito & $2020_{\mathrm{F}}$ & 54 & 4 & 4 & $0.07 \pm 1.41$ & $1-3$ & 0.006 \\
\hline Glomeritrema sp. & Specialist & Gills & Acapulco & $2011_{\mathrm{Ma}}$ & 76 & 11 & 55 & $0.72 \pm 10.84$ & $1-30$ & 0.034 \\
\hline & & & Acapulco & $2018_{J}$ & 84 & 4 & 5 & $0.06 \pm 1.15$ & $1-3$ & 0.022 \\
\hline & & & Acapulco & $2019_{\mathrm{M}}$ & 59 & 15 & 18 & $0.31 \pm 0.71$ & $1-3$ & 0.062 \\
\hline & & & Zapotalito & $2017_{\mathrm{F}}$ & 48 & 79 & 395 & $8.23 \pm 11.29 *$ & $1-58$ & 0.227 \\
\hline & & & Zapotalito & $2019 \mathrm{~J}$ & 40 & 33 & 35 & $0.88 \pm 2.14$ & $1-9$ & 0.084 \\
\hline & & & Zapotalito & $2020_{\mathrm{F}}$ & 54 & 48 & 159 & $2.94 \pm 7.32$ & $1-28$ & 0.082 \\
\hline $\begin{array}{l}\text { Gonocercella pacifica } \\
\text { Manter, } 1940^{\mathrm{f}}\end{array}$ & Generalist & Intestine & Zapotalito & $2020_{\mathrm{F}}$ & 54 & 2 & 1 & 0.02 & $1-1$ & 0.003 \\
\hline Lecithochirium microstomum & Generalist & Intestine & Acapulco & $2011_{\mathrm{Ma}}$ & 76 & 1 & 1 & 0.01 & $1-1$ & 0.004 \\
\hline Chandler, $1935^{\mathrm{f}}$ & & & Acapulco & $2012_{\mathrm{A}}$ & 76 & 1 & 3 & 0.04 & $1-3$ & 0.004 \\
\hline & & & Acapulco & $2018_{J}$ & 84 & 6 & 7 & $0.08 \pm 0.55$ & $1-2$ & 0.036 \\
\hline & & & Acapulco & $2018_{D}$ & 77 & 7 & 7 & $0.09 \pm 0.55$ & $1-2$ & 0.112 \\
\hline & & & Acapulco & $2019_{M}$ & 59 & 9 & 12 & $0.20 \pm 2.19$ & $1-6$ & 0.034 \\
\hline & & & Zapotalito & $2017_{\mathrm{F}}$ & 48 & 2 & 1 & 0.02 & $1-1$ & 0.006 \\
\hline & & & Zapotalito & $2017_{D}$ & 28 & 4 & 1 & 0.04 & $1-1$ & 0.030 \\
\hline & & & Zapotalito & $2019_{\mathrm{J}}$ & 40 & 8 & 5 & $0.13 \pm 0.58$ & $1-2$ & 0.019 \\
\hline & & & Zapotalito & $2020_{\mathrm{F}}$ & 54 & 4 & 2 & 0.04 & $1-1$ & 0.006 \\
\hline & & & L. Cárdenas & $2013_{\mathrm{N}}$ & 49 & 2 & 1 & 0.02 & $1-1$ & 0.01 \\
\hline & & & P. Vicente & $2018_{A p}$ & 64 & 2 & 1 & 0.02 & $1-1$ & 0.142 \\
\hline Prosorhynchoides cybii & Generalist & Intestine & Acapulco & $2011_{\mathrm{Ma}}$ & 76 & 21 & 50 & $0.66 \pm 3.79$ & $1-14$ & 0.068 \\
\hline Park, $1939^{\mathrm{f}}$ & & & Acapulco & $2012_{\mathrm{Ma}}$ & 19 & 37 & 48 & $2.53 \pm 7.43$ & $1-19$ & 0.082 \\
\hline & & & Acapulco & 2012 & 76 & 7 & 7 & $0.09 \pm 0.89$ & $1-3$ & 0.021 \\
\hline & & & Acapulco & $2018_{\mathrm{J}}^{\mathrm{A}}$ & 84 & 2 & 12 & $0.14 \pm 7.07$ & $1-11$ & 0.014 \\
\hline & & & Acapulco & $2018_{D}$ & 77 & 1 & 1 & 0.01 & $1-1$ & 0.022 \\
\hline & & & Acapulco & $2019_{\mathrm{M}}$ & 59 & 3 & 3 & $0.05 \pm 0.71$ & $1-2$ & 0.014 \\
\hline & & & Zapotalito & $2017_{\mathrm{F}}^{\mathrm{N}}$ & 48 & 23 & 19 & $0.40 \pm 1.56$ & $1-6$ & 0.066 \\
\hline & & & Zapotalito & $2019 \mathrm{~J}$ & 40 & 20 & 19 & $0.48 \pm 2.07$ & $1-6$ & 0.052 \\
\hline & & & Zapotalito & $2020_{\mathrm{F}}$ & 54 & $61 *$ & 173 & $3.20 \pm 5.68$ & $1-21$ & 0.104 \\
\hline & & & L. Cárdenas & $2013_{\mathrm{N}}$ & 49 & 45 & 69 & $1.41 \pm 3.18$ & $1-13$ & 0.13 \\
\hline & & & P. Vicente & $2018_{A p}$ & 64 & 5 & 3 & 0.05 & $1-1$ & 0.426 \\
\hline Monogenea & & & & & & & & & & \\
\hline Mexicotyle mexicana & Specialist & Gills & Acapulco & $2011_{\mathrm{Ma}}$ & 76 & 28 & 38 & $0.50 \pm 1.60$ & $1-8$ & 0.089 \\
\hline (Meserve, 1938) Lebedev, 1984 & & & Acapulco & $2012_{\mathrm{Ma}}$ & 19 & 63 & 52 & $2.74 \pm 2.84$ & $1-9$ & 0.141 \\
\hline & & & Acapulco & $2012_{\mathrm{A}}$ & 76 & 68 & 213 & $2.80 \pm 4.29$ & $1-26$ & 0.221 \\
\hline & & & Acapulco & 2018 & 84 & 31 & 51 & $0.61 \pm 1.40$ & $1-8$ & 0.186 \\
\hline
\end{tabular}


Table 1. Continued

\begin{tabular}{|c|c|c|c|c|c|c|c|c|c|c|}
\hline \multirow{11}{*}{$\begin{array}{l}\text { Scomberocotyle scomberomori } \\
\text { (Koratha, 1955) Hargis, } 1956\end{array}$} & & & & & & & & & & \\
\hline & & & Acapulco & $2018_{D}$ & 77 & 7 & 29 & $0.38 \pm 6.57$ & $1-13$ & 0.112 \\
\hline & & & Acapulco & $2019_{\mathrm{M}}$ & 59 & 39 & 47 & $0.80 \pm 1.43$ & $1-6$ & 0.158 \\
\hline & & & Zapotalito & $2017_{\mathrm{F}}$ & 48 & 73 & 157 & $3.27 \pm 4.12$ & $1-16$ & 0.210 \\
\hline & & & Zapotalito & $2017_{\mathrm{D}}$ & 28 & 25 & 10 & $0.36 \pm 0.79$ & $1-3$ & 0.207 \\
\hline & & & Zapotalito & $2019_{\mathrm{J}}$ & 40 & 53 & 55 & $1.38 \pm 1.77$ & $1-6$ & 0.136 \\
\hline & & & Zapotalito & $2020_{\mathrm{F}}$ & 54 & $91^{*}$ & 420 & $7.78 \pm 8.46^{*}$ & $1-46$ & 0.154 \\
\hline & & & L. Cárdenas & $2013_{N}$ & 49 & 45 & 66 & $1.35 \pm 2.47$ & $1-12$ & 0.128 \\
\hline & \multirow{3}{*}{ Specialist } & \multirow[t]{3}{*}{ Gills } & Acapulco & $2011_{\mathrm{Ma}}$ & 76 & 1 & 2 & 0.03 & $1-2$ & 0.004 \\
\hline & & & Acapulco & $2019_{\mathrm{M}}$ & 59 & 2 & 1 & 0.02 & $1-1$ & 0.007 \\
\hline & & & Zapotalito & $2020_{\mathrm{F}}$ & 54 & 13 & 12 & $0.22 \pm 1.25$ & $1-4$ & 0.022 \\
\hline \multirow{11}{*}{$\begin{array}{l}\text { Inoracocotyle crocea } \\
\text { MacCallum, } 1913\end{array}$} & \multirow[t]{11}{*}{ Specialist } & \multirow[t]{11}{*}{ Gills } & Acapulco & $2011_{M}$ & 76 & 62 & 240 & $3.16 \pm 5.75$ & $1-30$ & 0.199 \\
\hline & & & Acapulco & 2012 & 19 & 68 & 199 & $10.47 \pm 17.16$ & $1-55$ & 0.153 \\
\hline & & & Acapulco & 2012 & 76 & 82 & 345 & $4.54 \pm 5.18$ & $1-26$ & 0.263 \\
\hline & & & Acapulco & 2018 & 84 & 30 & 65 & $0.77 \pm 3.11$ & $1-14$ & 0.179 \\
\hline & & & Acapulco & $2018_{\mathrm{D}}$ & 77 & 9 & 11 & $0.14 \pm 0.53$ & $1-26$ & 0.157 \\
\hline & & & Acapulco & $2019_{M}$ & 59 & 25 & 155 & $2.63 \pm 20.36$ & $1-73$ & 0.103 \\
\hline & & & Zapotalito & $2017_{\mathrm{F}}$ & 48 & 60 & 253 & $5.27 \pm 10.71$ & $1-41$ & 0.174 \\
\hline & & & Zapotalito & $2017_{D}$ & 28 & 11 & 9 & $0.32 \pm 3.46$ & $1-73$ & 0.089 \\
\hline & & & Zapotalito & $2019_{\mathrm{J}}$ & 40 & 70 & 272 & $6.80 \pm 17.01$ & $1-72$ & 0.182 \\
\hline & & & Zapotalito & $2020_{\mathrm{F}}$ & 54 & 87 & 953 & $17.65 \pm 31.7 *$ & $1-186$ & 0.148 \\
\hline & & & L. Cárdenas & $2013_{N}$ & 49 & 71 & 266 & $5.43 \pm 10.22$ & $1-50$ & 0.20 \\
\hline \multicolumn{11}{|l|}{ Cestoda } \\
\hline Tetrafilidae ${ }^{\mathrm{f}}$ (larvae) & Generalist & Intestine & Acapulco & $2011_{\mathrm{Ma}}$ & 76 & 1 & 10 & 0.13 & $1-10$ & 0.004 \\
\hline \multicolumn{11}{|l|}{ Acanthocephala } \\
\hline Rhadinorhynchus cf. pristis & Generalist & Intestine & Acapulco & $2018_{J}$ & 84 & 2 & 3 & $0.04 \pm 0.71$ & $1-2$ & 0.014 \\
\hline (Rudolphi, 1802) Lühe, 1911 & & & Acapulco & $2019_{M}$ & 59 & 5 & 5 & $0.08 \pm 1.15$ & $1-3$ & 0.021 \\
\hline Nematoda & & & & & & & & & & \\
\hline Anisakis sp. & Generalist & Intestine & Acapulco & $2011_{\mathrm{Ma}}$ & 76 & 15 & 41 & $0.54 \pm 6.10$ & $1-21$ & 0.047 \\
\hline (larvae) & & & Acapulco & $2012_{\mathrm{Ma}}$ & 19 & $21^{*}$ & 34 & $1.79 \pm 12.40$ & $1-27$ & 0.047 \\
\hline & & & Acapulco & $2012_{\mathrm{A}}$ & 76 & 4 & 3 & 0.04 & $1-1$ & 0.013 \\
\hline & & & Acapulco & $2018_{\mathrm{J}}$ & 84 & 2 & 2 & 0.02 & $1-1$ & 0.014 \\
\hline & & & Acapulco & $2018_{D}$ & 77 & 3 & 2 & 0.03 & $1-1$ & 0.045 \\
\hline & & & Zapotalito & $2017_{\mathrm{F}}$ & 48 & 4 & 2 & 0.04 & $1-1$ & 0.012 \\
\hline & & & Zapotalito & $2020_{\mathrm{F}}$ & 54 & 7 & 5 & $0.09 \pm 0.50$ & $1-2$ & 0.013 \\
\hline & & & L. Cárdenas & $2013_{N}$ & 49 & 4 & 5 & $0.10 \pm 2.12$ & $1-4$ & 0.01 \\
\hline Hysterothylacium sp. & Generalist & Intestine & Acapulco & $2011_{\mathrm{Ma}}$ & 76 & 7 & 8 & $0.11 \pm 0.89$ & $1-3$ & 0.021 \\
\hline & & & Acapulco & $2012_{\mathrm{Ma}}$ & 19 & 21 & 10 & $0.53 \pm 1.73$ & $1-5$ & 0.047 \\
\hline & & & Acapulco & $2012_{\mathrm{A}}$ & 76 & 1 & 1 & 0.01 & $1-1$ & 0.004 \\
\hline & & & Acapulco & $2019_{\mathrm{M}}$ & 59 & 12 & 18 & $0.31 \pm 1.62$ & $1-6$ & 0.048 \\
\hline & & & Zapotalito & $2017_{\mathrm{F}}$ & 48 & 10 & 6 & $0.13 \pm 0.45$ & $1-2$ & 0.030 \\
\hline & & & Zapotalito & 2019 J & 40 & $40^{*}$ & 28 & $0.70 \pm 0.86$ & $1-3$ & 0.104 \\
\hline & & & Zapotalito & $2020_{\mathrm{F}}$ & 54 & 2 & 1 & 0.02 & $1-1$ & 0.003 \\
\hline & & & L. Cárdenas & $2013_{\mathrm{N}}^{\mathrm{r}}$ & 49 & 12 & 6 & 0.12 & $1-1$ & 0.03 \\
\hline Philometra sp. ${ }^{\mathrm{f}}$ & Generalist & Gonad & Acapulco & $2011_{\mathrm{Ma}}$ & 76 & 1 & 6 & 0.08 & $1-6$ & 0.004 \\
\hline & & & Acapulco & $2012_{\mathrm{Ma}}$ & 19 & 26 & 14 & $0.74 \pm 2.49$ & $1-6$ & 0.059 \\
\hline & & & Acapulco & $2012_{\mathrm{A}}$ & 76 & 1 & 1 & 0.01 & $1-1$ & 0.004 \\
\hline & & & Zapotalito & $2017_{\mathrm{F}}$ & 48 & 2 & 1 & 0.02 & $1-1$ & 0.006 \\
\hline & & & Zapotalito & $2020_{\mathrm{F}}$ & 54 & 2 & 4 & 0.07 & $1-4$ & 0.003 \\
\hline & & & L. Cárdenas & $2013_{N}$ & 49 & 2 & 1 & 0.02 & $1-1$ & 0.01 \\
\hline Procamallanus sp. ${ }^{\mathrm{f}}$ & Generalist & Intestine & Acapulco & $2012_{\mathrm{Ma}}$ & 19 & 11 & 10 & $0.53 \pm 1.41$ & $4-6$ & 0.024 \\
\hline & & & Acapulco & 2018 & 84 & 1 & 1 & 0.01 & $1-1$ & 0.007 \\
\hline & & & Zapotalito & $2020_{F}$ & 54 & 6 & 3 & 0.06 & $1-1$ & 0.009 \\
\hline Copepoda & & & & & & & & & & \\
\hline Caligus omissus & Specialist & Gills & Acapulco & $2011_{\mathrm{Ma}}$ & 76 & 26 & 30 & $0.39 \pm 0.76$ & $1-3$ & 0.085 \\
\hline Cressey et Cressey, $1980^{\mathrm{f}}$ & & & Acapulco & 2012 & 19 & $74 *$ & 24 & $1.26 \pm 1.14$ & $1-4$ & 0.165 \\
\hline & & & Acapulco & $2012_{\mathrm{A}}$ & 76 & $72^{*}$ & 112 & $1.47 \pm 1.56$ & $1-8$ & 0.233 \\
\hline & & & Acapulco & $2018_{\mathrm{J}}^{\mathrm{A}}$ & 84 & 55 & 70 & $0.83 \pm 0.62$ & $1-3$ & 0.330 \\
\hline & & & Acapulco & $2018_{D}$ & 77 & 27 & 31 & $0.40 \pm 0.75$ & $1-4$ & 0.470 \\
\hline & & & Acapulco & $2019_{\mathrm{M}}^{\mathrm{D}}$ & 59 & 37 & 31 & $0.53 \pm 0.73$ & $1-3$ & 0.151 \\
\hline & & & Zapotalito & $2017_{\mathrm{F}}$ & 48 & 58 & 82 & $1.71 \pm 4.14$ & $1-22$ & 0.168 \\
\hline & & & Zapotalito & $2017_{D}$ & 28 & 54 & 32 & $1.14 \pm 2.10$ & $1-9$ & 0.443 \\
\hline & & & Zapotalito & $2019_{\mathrm{J}}$ & 40 & 25 & 17 & $0.43 \pm 0.82$ & $1-3$ & 0.065 \\
\hline & & & Zapotalito & $2020_{\mathrm{F}}$ & 54 & 60 & 62 & $1.15 \pm 1.83$ & $1-10$ & 0.100 \\
\hline & & & L. Cárdenas & $2013_{\mathrm{N}}^{\mathrm{r}}$ & 49 & 51 & 43 & $0.88 \pm 1.70$ & $1-9$ & 0.15 \\
\hline & & & P. Vicente & 2018 & 64 & 5 & 3 & 0.05 & $1-10$ & 0.426 \\
\hline Ergasilus $\mathrm{sp} .{ }^{\mathrm{f}}$ & Generalist & Gills & L. Cárdenas & $2013_{N}$ & 49 & 2 & 1 & 0.02 & $1-1$ & 0.01 \\
\hline $\begin{array}{l}\text { Lernanthrophus cf. giganteus } \\
\text { Krøyer, } 1863^{\mathrm{f}}\end{array}$ & Generalist & Gills & Acapulco & $2011_{\mathrm{Ma}}$ & 76 & 3 & 2 & 0.03 & $1-1$ & 0.008 \\
\hline $\begin{array}{l}\text { Orbitacolax cf. aculeatus }{ }^{\mathrm{g}} \\
\text { Pillai, } 1962\end{array}$ & Generalist & Gills & Acapulco & $2012_{\mathrm{A}}$ & 76 & 3 & 3 & $0.04 \pm 0.71$ & $1-2$ & 0.008 \\
\hline
\end{tabular}


Table 1. Continued

\begin{tabular}{|c|c|c|c|c|c|c|c|c|c|c|}
\hline \multirow{6}{*}{$\begin{array}{l}\text { Cybicola buccatus } \\
\text { (Wilson, 1922) }^{\mathrm{f}}\end{array}$} & \multirow{6}{*}{ Specialist } & \multirow{6}{*}{ Gills } & Acapulco & $2018_{J}$ & 84 & 1 & 1 & 0.01 & $1-1$ & 0.007 \\
\hline & & & Acapulco & 2018 D & 77 & 1 & 1 & 0.01 & $1-1$ & 0.022 \\
\hline & & & Acapulco & $2018_{D}$ & 77 & 1 & 1 & 0.01 & $1-1$ & 0.022 \\
\hline & & & Acapulco & $2019_{M}$ & 59 & 12 & 7 & 0.12 & $1-1$ & 0.048 \\
\hline & & & Zapotalito & $2019^{1 / 4}$ & 40 & 5 & 3 & $0.08 \pm 0.71$ & $1-2$ & 0.013 \\
\hline & & & L. Cárdenas & $2013_{N}$ & 49 & 2 & 1 & 0.02 & $1-1$ & 0.01 \\
\hline \multicolumn{11}{|l|}{ Isopoda } \\
\hline Livoneca redmanii Leach, $1818^{\mathrm{f}}$ & ${ }^{\mathrm{f}}$ Generalist & Gills & Acapulco & 2018 & 84 & 2 & 2 & 0.02 & $1-1$ & 0.014 \\
\hline $\begin{array}{l}\text { Rocinela signata } \\
\text { Schioedte et Meinert, } 1879^{\mathrm{f}}\end{array}$ & Generalist & Gills & Zapotalito & $2019_{J}$ & 40 & 5 & 2 & 0.05 & $1-1$ & 0.013 \\
\hline
\end{tabular}

\section{Variation in infection level between locations and/or sampling years}

In eight of these widely distributed species (except for L. microstomum) prevalence varied significantly between locations and/or sampling years. Infection percentages were highest for M. mexicana $(\mathrm{G}=129.87, \mathrm{p}<0.05), T$. crocea $(\mathrm{G}=152.6, \mathrm{p}<0.05)$, Didymocilindrus $\mathrm{sp} .(\mathrm{G}=$ 136.46, $\mathrm{p}<0.05)$ and P. cybii $(\mathrm{G}=193.05, \mathrm{p}<0.05)$ in the Zapotalito $2020_{\mathrm{Feb}}$ sample, but were highest for Hysterothylacium sp. $(\mathrm{G}=83.87, \mathrm{p}<0.05)$ in the Zapotalito $2019_{\text {Jan }}$ sample (Table 1). The three remaining parasite species (Didymocystis sp. [G $=111.08, \mathrm{p}<0.05]$, Anisakis sp. [G $=42.32, \mathrm{p}<0.05]$, and C. omissus $[\mathrm{G}=108.28, \mathrm{p}<0.05])$ were more prevalent in Acapulco, mainly in the Acapulco $2012_{\text {Mar }}$ sample (Table 1). In contrast to the infection percentage results, only two parasite species (Hysterothylacium sp. [GLM: $\mathrm{F}_{2,674}=7.75, \mathrm{p}<0.01$ ] and $C$. omissus [GLM: $\left.\left.F_{2,674}=3.79, \mathrm{p}<0.05\right]\right)$ exhibited significant variation in mean abundance between sampling locations. However, the abundance of most species (except Anisakis sp.) varied between sampling years within the same location (GLM: $\mathrm{F}_{2,674}, \mathrm{p}<0.01$ ).

\section{Relationship between the host body size and the infection parameters}

The abundance of at least three species of parasites showed a positive or negative relationship with the host body size. Positive correlations were registered for the monogenean T. crocea in the Acapulco 2012 ( $\mathrm{rs}=0.257$, $\mathrm{p}<0.05)$ and L. Cárdenas $2013_{\mathrm{Nov}}(\mathrm{rs}=0.558, \mathrm{p}<0.05)$ samples, and for the digenean $P$. cybii in the samples of Acapulco 2011 ${ }_{\mathrm{Mar}}(\mathrm{rs}=0.574, \mathrm{p}<0.05)$, and Acapulco $2012_{\mathrm{Mar}}$ ( $\left.\mathrm{rs}=0.836, \mathrm{p}<0.05\right)$. Whereas abundance of the copepod $C$. omissus was negatively correlated with the host body size in the Acapulco $2012_{\mathrm{Mar}}$ ( $\left.\mathrm{rs}=-0.648, \mathrm{p}<0.05\right)$ and Zapotalito $2017_{\text {Jan }}$ ( $\mathrm{rs}=-0.686, \mathrm{p}<0.05$ ) samples.

Analyses of host size-classes by location and sampling year (Table 2) indicated that mean species richness and mean total intensity tended to increase from the smallest to largest size-classes $(<30 \mathrm{~cm}=1.92,5.1 ; 36-40 \mathrm{~cm}=$ $3.57,22.85 ;>40 \mathrm{~cm}=3.78,24.08)$. Positive correlations registered between total fish length and these infection parameters within some samples from two locations confirmed this trend (Table 2). Considering the twenty host size-classes constructed, the mean total length of each sizeclass correlated positively with the mean species richness $(\mathrm{rs}=0.480, \mathrm{p}<0.05)$ and mean total intensity $(\mathrm{rs}=0.639, \mathrm{p}$ $<0.01)$. No correlation was observed between the number of examined fish in each size-class and mean species richness ( $\mathrm{rs}=-0.303, \mathrm{p}>0.05)$, or mean total intensity ( $\mathrm{rs}=$ $-0.045, \mathrm{p}>0.05)$ indicating that the different sample sizes used in this analysis had no effect on the results.

\section{Host diet composition}

The dietary spectrum of $S$. sierra in the study area included nine items, with fish representing the highest proportion (88\%). Additonal prey items accounted only for $12 \%$ of the diet and included crab larvae, amphipods, penaeid shrimp larvae, mollusk larvae, isopods and stomatopods. Diet composition varied between locations and/ or sampling years $(t=6.62, p<0.01)$. For example, even though fish were the primary dietary component at all locations, diet composition was more variable at Zapotalito $2020_{\mathrm{Feb}}(\mathrm{H}=0.95)$ and Acapulco $2011_{\mathrm{Mar}}(\mathrm{H}=0.87)$ than at other locations such as Puerto Vicente $2018_{\text {Apr }}(\mathrm{H}=0.20)$. Feeding habits changed with fish body size; larger individuals $(>40 \mathrm{~cm})$ consumed higher percentages of fish. The diet was slightly more varied between individuals of 36-40 $\mathrm{cm}$ (mean $\mathrm{H}$ values: $<30 \mathrm{~cm}=0.47 ; 36-40 \mathrm{~cm}=0.61 ;>40$ $\mathrm{cm}=0.56$ ), and it was positively correlated with the mean parasite species richness among the twenty size-classes considered $(\mathrm{rs}=0.480, \mathrm{p}<0.05)$.

\section{Component community}

Parasite species richness recorded by location and/or sampling year (Table 3) varied widely from three (Puerto Vicente $2018_{\text {Apr }}$ ) to 16 (Zapotalito $\left.2020_{\mathrm{Feb}}\right)(\mathrm{t}=7.71, \mathrm{p}<$ $0.01)$. No correlation was observed between sample size and species richness at this level ( $\mathrm{rs}=0.293, \mathrm{p}>0.05)$. Total number of individual parasites ranged from seven $(\mathrm{P}$. Vicente $2018_{\text {Apr }}$ ) to 3,156 (Zapotalito $\left.2020_{\mathrm{Feb}}\right)(\mathrm{t}=8.58, \mathrm{p}$ $<0.05)$. Different parasite species numerically dominated the communities, although the monogenean $T$. crocea dominated up to five of the twelve examined communities. Shannon-Wiener diversity index values varied significantly from 0.48 (P. Vicente $2018_{\text {Apr }}$ ) to 1.20 (Zapotalito $\left.2020_{\mathrm{Feb}}\right)(\mathrm{t}=9.21, \mathrm{p}<0.01)$. Similarity between component communities was slightly low overall $(<70 \%$, Fig. 3$)$ and was higher at the qualitative level (mean $=65.6 \%)$ than at the quantitative level (mean $=32.5 \%$ ).

\section{Infracommunity}

Body size of $S$. sierra varied from $24.1 \pm 3.3 \mathrm{~cm}$ (Acapulco $2018_{\text {Dec }}$ ) to $47.2 \pm 4.3 \mathrm{~cm}$ (Acapulco 2012 ${ }_{\mathrm{Mar}}$ ) (Table 3 ), and differed between locations (ANOVA F ${ }_{3,618}=99.56$, $\mathrm{p}<0.01$ ) and sampling years (ANOVA $\mathrm{F}_{6,618} \stackrel{3,618}{=} 174.09, \mathrm{p}$ 


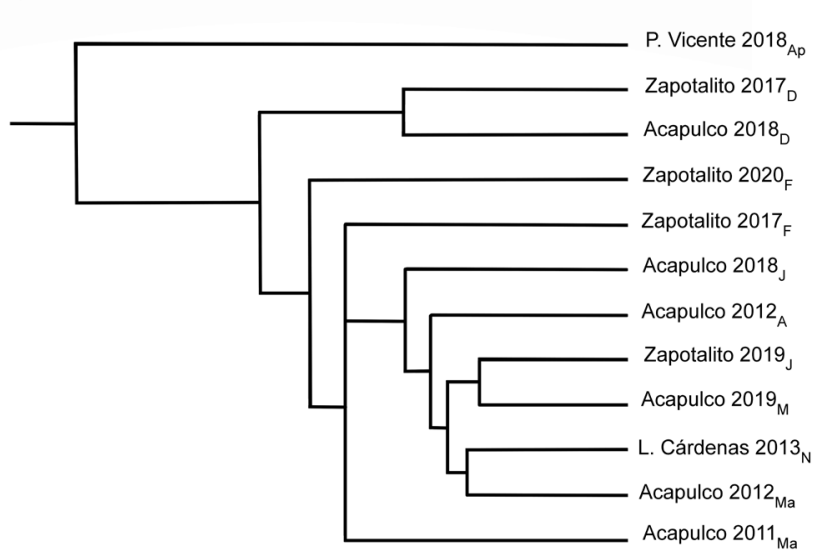

0. \% Similarity

50

100

Fig. 3. Similarity percentages (Bray-Curtis index) for parasite communities of Scomberomorus sierra (Jordan et Starks) between locations and sampling years.

$<0.01)$. No significant differences in size were observed between male and female fish ( $p>0.05)$.

Mean parasite species richness ranged from 1.0 to 5.6 \pm 1.5 and mean number of individual parasites from 1.0 to $43.9 \pm 31.5$ (Table 3). Brillouin diversity index $\left(\mathrm{H}^{\prime}\right)$ values varied from $0.13 \pm 0.30$ to $1.46 \pm 0.50$. Values for all three parameters (mean number of species [GLM: $F_{2,618}=37.62$, $\mathrm{p}<0.01$ ], mean number of parasite individuals [GLM: $\mathrm{F}_{2,618}$ $=44.01, \mathrm{p}<0.01]$ and mean diversity $\left(\mathrm{H}^{\prime}\right)$ [GLM: $\mathrm{F}_{2,618}=$ 27.46, $\mathrm{p}<0.01$ ]) were highest in the Zapotalito $2020_{\mathrm{Feb}}$ sample (Table 3). Infracommunity index values (ICI) indicated that the monogeneans $M$. mexicana and T. crocea, the didymozoid Didymocilindrus sp. and the copepod C. omissus had the highest number of double or multiple infections with other parasite species (ICI mean $>0.15$ ), at almost all locations or in nearly all sampling years (Table 1).

\section{Multivariate analyses}

The PCA applied to identify the influence of biotic and abiotic factors on parasite infracommunity structure (Fig. 4), the KMO (0.43) and the Bartlett's tests $\left(\chi^{2}=4,860.2\right.$; d.f. $=66, \mathrm{p}<0.001)$, identified a sufficient relationship between the number of studied variables and the sample sizes, thus confirming the PCA's relevance. The first two component variables generated by the model explained $55 \%$ of total variance; they contributed $32 \%$ (eigenvalue $=$ 3.78 ) and $24 \%$ (eigenvalue $=2.82$ ), respectively. The first variable suggests that the richest and most diverse infracommunities among all the sampled locations were characterised by a significant number of didymozoid species. These infracommunities were mainly recorded in larger hosts. However, these infracommunities' richness and diversity varied between climatic seasons and locations. For example, a positive correlation for season and a negative one for sampling location variables (Table 4) suggested that the richest and most diverse infracommunities were collected from Zapotalito $2020_{\mathrm{Feb}}$ during the dry season (Table 3).

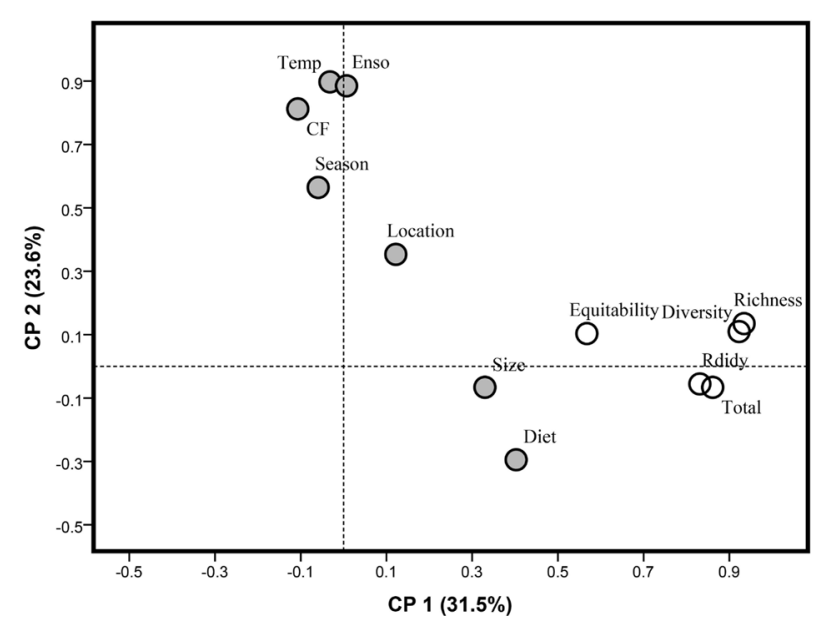

Fig. 4. Scatter plot of Principal Component Analysis (PCA) of factors that influence the species richness and diversity of the parasite infracommunities in Scomberomorus sierra (Jordan et Starks) from Mexican Pacific coast. Predictor variables ( $\bigcirc$ ): Location $=$ collection location, Season $=$ climatic season $($ dry $/$ rainy), Temp $=$ surface temperature values, Enso $=$ multivariate El Niño index, Diet $=$ host diet variety, Size $=$ host body size (total length), $\mathrm{CF}=$ Fulton's condition factor, Rdidy = number of didymozoid species. Infracommunity parameters $(\bigcirc)$ : Richness $=$ number of parasite species per infracommunity, Diversity $=$ Brillouin diversity index values, Equitability $=$ species evenness, Total $=$ total number of parasites per infracommunity.

The model built to identify locations via differences in parasite community species composition showed the first two discriminant variables to explain $97 \%$ of total variance, contributing $54 \%$ (eigenvalue $=0.705$ ) and $43 \%$ (eigenvalue $=0.563)$, respectively. A significant overall group effect was observed (Wilks' lambda $=0.361, \mathrm{p}<0.001$ ). Individual fish were mainly distributed along the first axis (Fig. 5). Dimensionality tests showed that the four studied locations were significantly separate in both dimensions $\left(\chi^{2}=190.5\right.$, d.f. $=$ $24, \mathrm{p}<0.001$ ) (Fig. 5). Each fish was correctly assigned to one of the four sampling locations at a $73 \%$ accuracy, more than twice the $26 \%$ produced by chance alone.

Of the 14 selected component species (i.e. prevalence $>10 \%$ ), only six were accepted by the model based on their lower Wilks' lambda values. The high prevalences of the monogenean M. mexicana, the didymozoid Glomeritrema sp. and the copepod $C$. omissus effectively assigned their hosts to Zapotalito (Table 5). The higher abundances observed for the digeneans $P$. cybii, Didymocilindrus sp. and Didymocystis sp. were characteristic of hosts from Lázaro Cárdenas. High prevalence for $C$. omissus also helped to separate out most $(68 \%)$ of the hosts from Acapulco. None of the component species was effective at assigning fish hosts to Puerto Vicente (Table 5).

\section{DISCUSSION}

The parasite communities of Scomberomorus sierra were characterised by numerical dominance of ectoparasites. Parasite community structure and species composition varied between locations, seasons and sampling years. Despite the presence of a distinctive set of host-specialist parasites (monogeneans, didymozoids and copepods), sim- 
Table 2. Relationships between host body size and the infection parameters of metazoan parasites communities in Scomberomorus sierra (Jordan et Starks) from Mexican Pacific coasts (Zapotalito, Oaxaca; Acapulco and Puerto Vicente, Guerrero; and Lázaro Cárdenas, Michoacán).

\begin{tabular}{|c|c|c|c|c|c|c|c|c|c|c|}
\hline Location & $\begin{array}{l}\text { Sample/ } \\
\text { size-class }\end{array}$ & $\begin{array}{l}\text { Examined } \\
\text { fish }\end{array}$ & No. infected & $\begin{array}{c}\mathrm{CV}^{\mathrm{a}} \\
\%\end{array}$ & Diet & $\begin{array}{l}\text { Mean } \\
\text { richness }\end{array}$ & $\begin{array}{c}\text { Total } \\
\text { prevalence }\end{array}$ & $\begin{array}{l}\text { Mean total } \\
\text { intensity }\end{array}$ & $\begin{array}{l}\text { Fish length } \\
\text { vs. richness }\end{array}$ & $\begin{array}{c}\text { Fish length } \\
\text { vs. intensity } \\
\end{array}$ \\
\hline \multirow[t]{14}{*}{ Acapulco } & Mar 2011 & & & & & & & & & \\
\hline & $>40 \mathrm{~cm}$ & 76 & 75 & 7.6 & 0.87 & $3.1 \pm 1.5$ & 98.6 & $28.0 \pm 24.5$ & $0.467^{*}$ & -0.021 \\
\hline & Mar 2012 & & & & & & & & & \\
\hline & $>40 \mathrm{~cm}$ & 19 & 19 & 10.7 & 0.53 & $4.5 \pm 1.9$ & 100 & $32.9 \pm 23.9$ & 0.003 & -0.151 \\
\hline & Aug 2012 & & & & & & & & & \\
\hline & $<35 \mathrm{~cm}$ & 11 & 11 & 8.4 & 0.46 & $3.4 \pm 0.8$ & 100 & $11.9 \pm 8.2$ & -0.094 & -0.545 \\
\hline & $36-40 \mathrm{~cm}$ & 28 & 28 & 3.9 & 0.59 & $3.3 \pm 1.1$ & 100 & $14.6 \pm 9.8$ & -0.142 & -0.001 \\
\hline & $>40 \mathrm{~cm}$ & 37 & 37 & 6.3 & 0.50 & $2.9 \pm 0.7$ & 100 & $14.6 \pm 10.4$ & $0.405 *$ & 0.247 \\
\hline & Jan 2018 & & & & & & & & & \\
\hline & $\begin{array}{l}<35 \mathrm{~cm} \\
\text { Dec } 2018\end{array}$ & 84 & 73 & 10.1 & 0.79 & $1.9 \pm 1.0$ & 86.9 & $4.5 \pm 5.3$ & 0.100 & 0.001 \\
\hline & $\begin{array}{l}<35 \mathrm{~cm} \\
\text { May } 2019\end{array}$ & 77 & 35 & 13.8 & 0.42 & $1.3 \pm 0.7$ & 45.4 & $2.9 \pm 5.0$ & $0.439 *$ & $0.419 *$ \\
\hline & $<35 \mathrm{~cm}$ & 29 & 24 & 4.5 & 0.50 & $2.0 \pm 1.0$ & 82.8 & $5.2 \pm 5.7$ & 0.328 & $0.454^{*}$ \\
\hline & $36-40 \mathrm{~cm}$ & 15 & 14 & 4.1 & 0.53 & $4.5 \pm 1.9$ & 93.3 & $17.0 \pm 17.0$ & -0.222 & -0.116 \\
\hline & $>40 \mathrm{~cm}$ & 15 & 11 & 4.8 & 0.68 & $3.2 \pm 2.0$ & 73.3 & $14.1 \pm 23.6$ & -0.349 & -0.468 \\
\hline \multirow[t]{10}{*}{ Zapotalito } & Feb 2017 & & & & & & & & & \\
\hline & $36-40 \mathrm{~cm}$ & 10 & 10 & 13.6 & & $3.4 \pm 1.3$ & 100 & $10.7 \pm 6.4$ & $0.484 *$ & 0.142 \\
\hline & $\begin{array}{l}>40 \mathrm{~cm} \\
\text { Dec } 2017\end{array}$ & 38 & 38 & 6.46 & & $3.5 \pm 1.3$ & 100 & $23.2 \pm 21.73$ & 0.116 & 0.123 \\
\hline & $36-40 \mathrm{~cm}$ & 17 & 12 & 8.7 & & $1.7 \pm 0.6$ & 70.6 & $5.1 \pm 3.2$ & -0.085 & -0.115 \\
\hline & $>40 \mathrm{~cm}$ & 11 & 9 & 2.2 & & $1.6 \pm 0.7$ & 81.8 & $2.2 \pm 1.6$ & 0.038 & 0.139 \\
\hline & Jan 2019 & & & & & & & & & \\
\hline & $>40 \mathrm{~cm}$ & 40 & 40 & 4.6 & 0.61 & $3.8 \pm 1.6$ & 100 & $17.5 \pm 18.9$ & -0.086 & 0.019 \\
\hline & Feb 2020 & & & & & & & & & \\
\hline & $36-40 \mathrm{~cm}$ & 11 & 11 & 4.3 & & $5.9 \pm 1.0$ & 100 & $77.5 \pm 48.4$ & -0.389 & -0.355 \\
\hline & $>40 \mathrm{~cm}$ & 43 & 43 & 4.4 & & $5.9 \pm 1.6$ & 100 & $55.6 \pm 43.1$ & 0.028 & -0.292 \\
\hline \multirow[t]{3}{*}{$\begin{array}{l}\text { Lázaro } \\
\text { Cárdenas }\end{array}$} & Nov 2013 & & & & & & & & & \\
\hline & $36-40 \mathrm{~cm}$ & 21 & 19 & 5.1 & & $3.9 \pm 1.3$ & 90.5 & $19.6 \pm 19.5$ & -0.370 & -0.376 \\
\hline & $>40 \mathrm{~cm}$ & 28 & 27 & 14.8 & & $3.6 \pm 1.8$ & 96.4 & $17.6 \pm 16.0$ & 0.041 & 0.009 \\
\hline \multirow[t]{2}{*}{$\begin{array}{l}\text { Puerto } \\
\text { Vicente }\end{array}$} & Apr 2018 & & & & & & & & & \\
\hline & $<35 \mathrm{~cm}$ & 64 & 7 & 5.2 & 0.20 & 1 & 10.9 & 1 & & \\
\hline
\end{tabular}

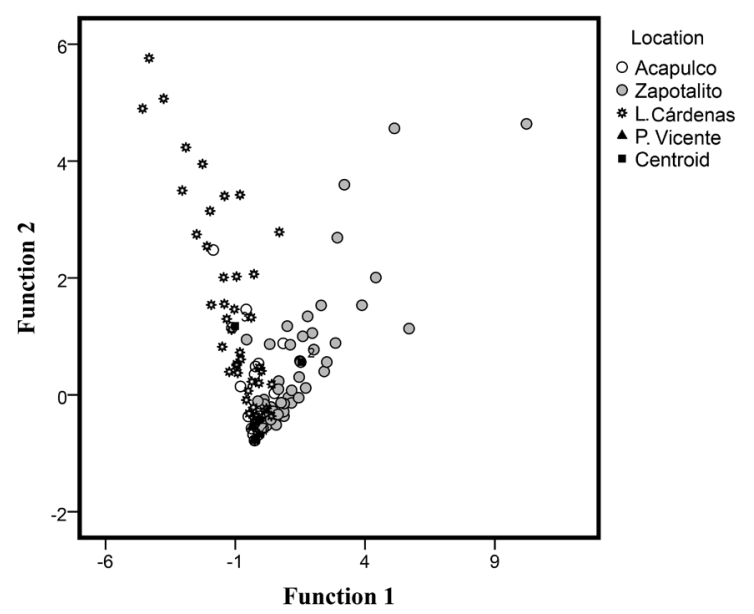

Fig. 5. Graphic of multivariate discriminant analyses for parasite communities of Scomberomorus sierra from four locations. The symbols represent each one of the sampling locations. Centroid $=$ mean group.

ilarity between the component parasite communities was generally low. Interannual or local variations in some biotic and abiotic environmental factors are possible causes of the observed variations in S. sierra parasite community structure and species composition. Ecological factors were therefore considered more important than phylogenetic aspects (host phylogeny) in structuring parasite communities.

\section{Parasite community species composition}

The overall dominance pattern of the main metazoan parasite taxa recorded in S. sierra was Digenea $>$ Copepoda $>$ Nematoda $>$ Monogenea $>$ Isopoda $>$ Cestoda $=$ Acanthocephala. Fourteen (seven helminths and seven crustaceans) of the 24 recorded parasite taxa are new geographical records for S. sierra on Mexico's Pacific coast (Table 1). Ectoparasites (three monogeneans, three didymozoids, five copepods and two isopods) numerically dominated the parasite communities, accounting for $82 \%$ of the total number of individual parasites recovered. Monogeneans are often transmitted directly between individual hosts through contact, exhibit high host-specificity, have a direct life cycle and can reproduce in a wide range of temperatures (Mendlová and Šimková 2014). The monogenean Thoracocotyle crocea was the dominant parasite in as many as five of the 12 examined parasite communities. This species has been recorded in all 18 species of Scomberomorus Lacepède, 


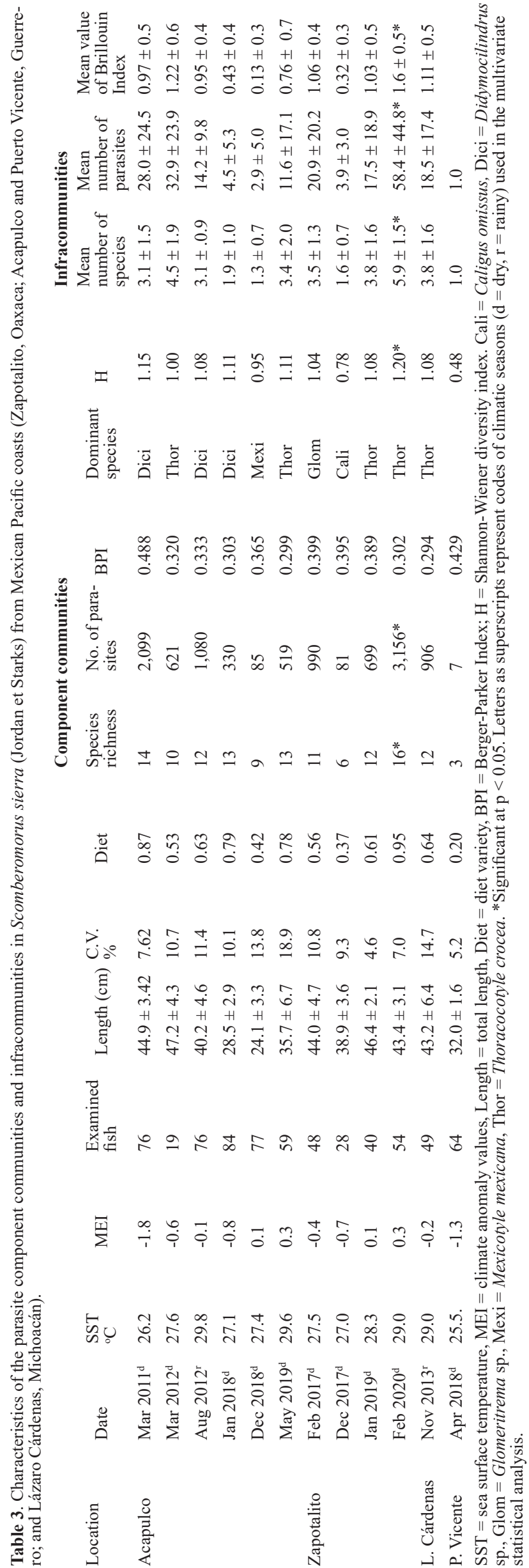

from both the Pacific and Atlantic coasts of the Americas (Hayward and Rohde 1999).

Didymozoids are also considered to be highly host-specific; this parasite group mainly parasitises tropical and subtropical scombrids and is rarely found in freshwater fish species (Madhavi and Ram 2000, Mele et al. 2012). Five didymozoid species have been reported in S. japonicus from Peru (Cruces et al. 2014) and six species (including the five didymozoid species reported here) have been reported in S. sierra from another location on Mexico's Pacific coast (Bárcenas 2019).

\section{Variation in parasite infection levels between sampling years and/or locations}

Temporal and spatial variation in infection levels were identified in as many as eight $(33 \%$ of the total number of species) of the parasite species collected from $S$. sierra. This is a common pattern in many marine parasite species (Poulin 2006, Villalba-Vásquez et al. 2018, Miranda-Delgado et al. 2019, Violante-González et al. 2020). Five of these eight were intestinal helminths, suggesting that these variations may be explained by changes in host diet between locations and/or sampling years, and the consequent availability of prey harbouring infective stages (Luque at al. 2004, Alves and Luque 2006). Although S. sierra is considered to have a specialised piscivorous diet (Collette and Nauen 1983), feeding mainly on small forage fish, dietary analysis has shown that along the Pacific coast of Mexico it is an opportunistic predator (Moreno-Sánchez et al. 2011). It can feed on prey that is abundant during a specific season or at a specific location. The present results showed it to have a more variable diet at some locations and/or in different sampling years. For example, the digeneans Didymocilindrus sp. and Prosorhynchoides cybii were most prevalent in the Zapotalito $2020_{\mathrm{Feb}}$ sample (Table 1).

Host diet is an important factor in the structuring of intestinal parasite communities in marine fish (Sasal et al. 1997, Luque et al. 2004, Villalba-Vásquez et al. 2018). However, the population density and gregarious behaviour (school training) of S. sierra (see Collette and Nauen 1983) may better explain the temporal and spatial variation observed in three ectoparasite species: Mexicotyle mexicana, T. crocea and Caligus omissus. Formation of large schools, as occurs in S. sierra, facilitates parasite transmission, particularly of those with a direct transmission cycle such as monogeneans and copepods (Sasal et al. 1997, Luque et al. 2004).

Variations in abiotic factors such as local fluctuations in surface temperature are known to affect many ecological processes, including the productivity of food webs, and the transmission rates of many trophically transmitted parasites due to population increase or decreases in potential intermediate or final hosts (Oliva et al. 2008, Miranda-Delgado et al. 2019). Trophically-transmitted parasites have free-living stages that are affected by the same oceanographic physical conditions (e.g. temperature, salinity, currents) that affect the larval stages of other marine organisms, including those of their hosts (Lohmus and Bjorklund 2015). 
Table 4. Summary of the Principal Component Analysis (PCA) on biotic and abiotic factors that influence the diversity and species richness of the infracommunities of Scomberomorus sierra (Jordan et Starks). The first and second principal component axes are indicated by PC1 and PC2, respectively. Variables that contribute to the total explained variance in each PC are indicated by an asterisk. Eigenvalues, percentage of variance and accumulative variance are shown at the end of the table. Communality = total influence of the variable with respect to all other associated variables. Uniqueness = percentage of variability that is not predicted by the variable in the model. MSA = Measure of Sampling Adequacy.

\begin{tabular}{|c|c|c|c|c|c|}
\hline & & & & Communality & Uniqueness \\
\hline Variables & $\mathrm{PC} 1$ & PC2 & MSA & $\mathrm{R}^{2}$ & $\%$ not predicted \\
\hline Richness & $0.934 *$ & 0.135 & 0.781 & 0.891 & 0.109 \\
\hline Diversity & $0.923 *$ & 0.110 & 0.822 & 0.864 & 0.136 \\
\hline Total & $0.861^{*}$ & -0.066 & 0.705 & 0.746 & 0.254 \\
\hline Rdidy & $0.831 *$ & -0.055 & 0.891 & 0.694 & 0.306 \\
\hline Equitability & $0.568^{*}$ & 0.341 & 0.652 & 0.333 & 0.667 \\
\hline Diet & $0.403 *$ & -0.295 & 0.190 & 0.249 & 0.751 \\
\hline Size & $0.330 *$ & -0.066 & 0.419 & 0.113 & 0.887 \\
\hline Temp & -0.032 & $0.898^{*}$ & 0.289 & 0.807 & 0.193 \\
\hline Enso & 0.007 & $0.885^{*}$ & 0.280 & 0.783 & 0.217 \\
\hline $\mathrm{CF}$ & -0.107 & $0.812^{*}$ & 0.958 & 0.672 & 0.328 \\
\hline Season & -0.059 & $0.565^{*}$ & 0.133 & 0.322 & 0.678 \\
\hline Location & 0.122 & $-0.353 *$ & 0.094 & 0.140 & 0.860 \\
\hline Eigenvalue & 3.78 & 2.82 & & & \\
\hline$\%$ total variance & 31.50 & 23.60 & & & \\
\hline Accumulative variance & 31.50 & 55.10 & & & \\
\hline
\end{tabular}

The PCA results (Fig. 4, Table 4) suggest that interannual variations in local environmental factors (i.e. temperature, climate season and ENSO index) had a substantial effect on parasite infracommunity structure. Some studies carried out in Acapulco Bay indicate that slight changes in the temperature and salinity parameters can be enough to generate notable changes in species composition of copepod communities throughout the year (Rojas-Herrera et al. 2016). For example, decreases in water temperature in this Bay during the first months of the year have been associated with increases in the cyclopoid and harpacticoid copepods population (Rojas-Herrera et al. 2016), both important intermediate hosts for intestinal helminths (Rohde 2005, Miranda-Delgado et al. 2019).

\section{Component communities}

Parasite species richness at the component level in $S$. sierra (3 to 16 parasite taxa; Table 3) was similar to the thirteen species reported for this host species at another location on Mexico's Pacific coast (Bárcenas 2019). It was also similar to the species richness in other species of Scomberomorus, such as $S$. commerson (Lacepède) (14 species), S. brasiliensis (Collette, Russo et Zavala-Camin) (7 species) and $S$. maculatus (Mitchill) (5 species) (Lester et al. 2001, Alves and Luque 2006, Aguirre-Macedo et al. 2007). Hosts with a broad geographical distribution are exposed to large numbers of parasite species because they interact with myriad intermediate host species throughout their distribution (Sasal et al. 1997). The present data support this hypothesis since a combined total of 24 parasite species taxa were recorded in S. sierra at the four sampled locations during the study period (Table 1).
Similarity in species composition between the parasite communities (Fig. 3) was generally low $(<70 \%)$ at both the qualitative and quantitative levels compared to parasite community similarity values reported for other marine fish from the Pacific coast of Mexico, namely $76 \%$ in Parapsettus panamensis (Steindachner) (Villalba-Vásquez et al. 2018), 79\% in Caranx caballus (Günther) (Gallegos-Navarro et al. 2018) and 85\% in Oligoplites altus (Günther) (Santos-Bustos et al. 2018). Perhaps the low similarity values observed in $S$. sierra were due to the fact that $50 \%$ of the identified parasite taxa (12 species: seven helminths and five crustaceans) were recorded in only one or two locations or in very few sampling years (Table 1). Local and interannual variations in biotic (e.g. diet, body size, availability of larva-infected prey) and/or abiotic environmental factors (Luque et al. 2004, Timi et al. 2010), may have caused the low similarity between the studied parasite communities of $S$. sierra.

\section{Infracommunities}

At the infracommunity level mean species richness and diversity values in $S$. sierra (mean number of species $=1$ to 5.6 , mean diversity $=0.13$ to 1.46 ; Table 3 ) were also similar to those reported for other parasite infracommunities of predatory marine fish on the Pacific coast of Mexico (Gallegos-Navarro et al. 2018, Violante-González et al. 2020). However, they were lower than those reported in another scombrid fish, Euthynnus lineatus (mean number of species $=4.7$ to 8.2 , mean diversity $=1.31$ to 1.94$)$ (Miranda-Delgado et al. 2019).

Several biotic and abiotic factors are known to be important determinants of species richness and diversity in the parasite communities of marine fish (Luque et al. 2004, 
Table 5. Discriminant analysis classification showing the numbers and percentages of the Scomberomorus sierra (Jordan et Starks) classified in each sampling location (rows correspond to group memberships). The bottom of the table shows the matrix of classification coefficient values for parasites that allowed differentiation between sampling locations; asterisks indicate the importance of each parasite species in distinguishing among locations.

\begin{tabular}{|c|c|c|c|c|c|}
\hline Location & Acapulco & Zapotalito & L. Cárdenas & P. Vicente & Percent \\
\hline Acapulco & 57 & 1 & 3 & 23 & 67.9 \\
\hline Zapotalito & 13 & 30 & 1 & 4 & 62.5 \\
\hline L. Cárdenas & 12 & 0 & 28 & 9 & 57.1 \\
\hline P. Vicente & 3 & 0 & 0 & 61 & 95.3 \\
\hline \multicolumn{6}{|l|}{ Parasite } \\
\hline Mexicotyle mexicana & 0.136 & $0.538^{*}$ & 0.261 & 0.000 & \\
\hline Prosorhynchoides cybii & 0.029 & 0.114 & $0.527^{*}$ & 0.022 & \\
\hline Didymocilindrus sp. & 0.094 & 0.054 & $0.273 *$ & -0.001 & \\
\hline Glomeritrema sp. & -0.017 & $0.287^{*}$ & -0.040 & 0.000 & \\
\hline Didymocystis sp. & -0.063 & -0.077 & $0.457 *$ & -0.001 & \\
\hline Caligus omissus & $0.257^{*}$ & $0.518^{*}$ & 0.174 & 0.014 & \\
\hline
\end{tabular}

Miranda-Delgado et al. 2019). The PCA results (Fig. 4) and ICI values (Table 1) indicated that infracommunity species richness and diversity in S. sierra depend heavily on the occurrence of a distinctive set of specialist parasite species (M. mexicana, T. crocea, Didymocilindrus sp., Didymocystis sp., Glomeritrema sp. and C. omissus). Host phylogeny (i.e. phylogenetic affinities) should therefore be considered as a crucial determinant of parasite infracommunity structure (Poulin et al. 2011, Krasnov et al. 2012) in S. sierra.

However, species frequency varied between sampling years and locations (Table 1). Several studies indicate that temporal variations in parasite infracommunity species composition can be attributed to variations in the prevalence and/or abundance of dominant taxa (Garcias et al. 2001, Villalba-Vásquez et al. 2018). As suggested above, fluctuations in the infection levels of some specialist parasites (such as didymozoid species) can be attributed mainly to changes in $S$. sierra feeding behaviour or foraging habitat over time and between locations.

Host body size was also an important structuring factor in the studied parasite infracommunities (Table 2, Fig. 4). Positive correlations between the total length and abundance of some parasite species, as well as the mean species richness and mean total intensity within each sample and across the locations or sampling year (Table 2), showed that this host trait was also an important factor in the structuring of parasite infracommunities of S. sierra. In the PCA results this variable made also a sizable contribution to the final built model (Table 4). In marine fish populations, the body size has proved to be the main predictor of the presence of a particular parasite species, of total abundance, and of species richness (Timi and Poulin 2003, Luque et al. 2004, Luque and Poulin 2008, Villalba-Vásquez et al. 2018). A large body size can facilitate the ectoparasite attachment; in addition, larger individuals tend to ingest greater quantities of food and are older and thus have had more time to accumulate parasites than smaller individuals (Timi and Poulin 2003, Luque et al. 2004, Luque and Poulin 2008).

The predatory habits of $S$. sierra promote accumulation of trophically-transmitted parasite species throughout this host's long life span. Predatory fish such as $S$. sierra are larger than their prey (Collette and Nauen 1983) and thus offer larger target areas for contact-transmitted parasites such as ectoparasites.

The discriminant analysis (Fig. 5, Table 5) identified at least six parasite species (M. mexicana, Didymocilindrus sp., Didymocystis sp., Glomeritrema sp., P. cybii and $C$. omissus) as biological tags useful in differentiating between S. sierra stocks along Mexico's southern Pacific coast. Parasites are well established as biological tags for distinguishing between stocks of the same fish species (Timi 2007, Poulin and Kamiya 2015), but the parasite fauna method has received only limited use in Mexico's fisheries (Violante-González et al. 2016).

Overall, the parasite communities of S. sierra were characterised by high ectoparasite species richness, with monogeneans and some didymozoids being numerically dominant. Parasite community structure and species composition varied between locations, seasons and sampling years. These variations were probably due to factors such as host traits (e.g. feeding behaviour and body size), presence of a set of distinctive parasite species (i.e. specialist parasite species), and possible interlocation differences in environmental factors and infected prey availability.

Acknowledgements. The authors wish to thank to students of the Marine Ecology Academic Unit (UAGro) for their assistance with field and laboratory work. Comments from two anonymous reviewers helped to substantially improve this manuscript.

\section{REFERENCES}

Aguirre-Macedo M.L., Vidal-Martínez V.M., GonzálezSolís D., Caballero P.I. 2007: Helminth communities of four commercially important fish species from Chetumal Bay, México. J. Helminthol. 80: 1-14.
Aguirre-Villaseñor H., Morales-Bojórquez E., Moran-Angulo R.E., Madrid-Vera J., Valdez-Pineda M.C. 2006: Biological indicators for the Pacific sierra (Scomberomorus sierra) fishery in the southern Gulf of California, México. Cienc. Mar. 32: 471-484. 
Alves D.R., Luque J.L. 2006: Ecologia das comunidades de metazoários parasitos de cinco espécies de escombrídeos (Perciformes: Scombridae) do litoral do Estado do Rio de Janeiro, Brasil. Rev. Bras. Parasitol. Vet. 15: 167-181.

BÁRCENAS N.Y. 2019: Variación anual de la estructura de la comunidad de helmintos de Scomberomorus sierra (Actinopterigii: Scombridae) en Mazatlán, México. Ph. D. Thesis. Instituto de Biología Sistemática. Universidad Nacional. Autónoma de México, Mexico City, 92 pp.

Bush A.O., Ано J.M., Kennedy C.R. 1990: Ecological versus phylogenetic determinants of helminth parasite community richness. Evol. Ecol. 4: 1-20.

Bush A.O., Lafferty K.D., Lotz J.M., Shostak A.W. 1997: Parasitology meets ecology on its own terms: Margolis et al. revisited. J. Parasitol. 83: 575-583.

Collette B.B., Nauen C.E. 1983: FAO Species Catalogue. Vol. 2. Scombrids of the World. An Annotated and Illustrated Catalogue of Tunas, Mackerels, Bonitos and Related Species Known to Date.FAO Fishery Synopsis, FAO, Rome, 125: $137 \mathrm{pp}$.

Cruces C., Chero J., Iannacone J., Diestro A., Sáez G., AlVARIÑo L. 2014: Metazoan parasites of 'chub mackerel' Scomber japonicus Houttuyn, 1782 (Perciformes: Scombridae) at the port of Chicama, La Libertad, Perú. Neotrop. Helminthol. 8: 357-381.

Gallegos-Navarro Y., Violante-González J., Monks S., García-Ibáñez S., Rojas-Herrera A.A., Pulido-Flores G., Rosas-Acevedo J.L. 2018: Factors linked to temporal and spatial variation in the metazoan parasite communities of green jack Caranx caballus (Günther 1868) (Pisces: Carangidae) from the Pacific coast of Mexico. J. Nat. Hist. 52: 2573-2590.

Garcias F., Mendoza R., George-Nascimento M. 2001: Between-years variation in infracommunities of metazoan parasites of the croaker Cilus gilberti (Pisces: Sciaenidae) from Chile. Rev. Chil. Hist. Nat. 74: 833-840.

Hay Ward C.J., Rohde K. 1999: Revision of the monogenean subfamily Thoracocotylinae Price, 1936 (Polyopisthocotylea: Thoracocotylidae), with the description of a new species of the genus Pseudothoracocotyla Yamaguti, 1963. Syst. Parasitol. 44: 157-169.

Henríquez V., González M.T. 2012: Patterns of variation in parasite component communities and infracommunities of a littoral fish species from the northern coast of Chile. J. Helminthol. 88 $88-96$.

Krasnov B.R., Mouillot D., Khokhlova I.S., Shenbrot G.I., Poulin R. 2012: Compositional and phylogenetic dissimilarity of host communities drives dissimilarity of ectoparasite assemblages: geographical variation and scale-dependence. Parasitology 139: 338-347.

Lester R.J.G., Thompson M.H., Barker S.C. 2001: Movement and stock structure of narrow-barred Spanish mackerel as indicated by parasites. J. Fish Biol. 59: 833-842.

Lima-Junior S.E., Goitein R. 2001: A new method for the analysis of fish stomach contents. Acta Scientiarum 23: 421-424.

Lohmus M., BJörkLund M. 2015: Climate change: what will it do to fish-parasite interactions? Biol. J. Linn. Soc. 116: 397-411.

Luque J.L., Mouillot D., Poulin R. 2004: Parasite biodiversity and its determinants in coastal marine teleost fishes of Brazil. Parasitology 128: 671-682.

LuQUe J.L., POulin R. 2008: Linking ecology with parasite diversity in Neotropical fishes. J. Fish Biol. 72: 189-204.

MadHavi R., Ram B.K. 2000: Community structure of helminth parasites of the tuna, Euthynnus affinis, from the Visakhapatnam coast, Bay of Bengal. J. Helminthol. 74: 337-342.

Mele S., Macias D., Gomez-Vives M.J., Garippa G., Alemany F., Merella P. 2012: Metazoan parasites on the gills of the skipjack tuna Katsuwonus pelamis (Osteichthyes: Scombridae) from the Alboran Sea (western Mediterranean Sea). Dis. Aquat. Org. 97: 219-225.
Mendlová M., Šımková A. 2014: Evolution of host specificity in monogeneans parasitizing African cichlid fish. Parasit. Vect. 7: $1-14$.

Miranda-Delgado J.E., Violante-González J., Monks S., Rojas-Herrera A.A., García-Ibáñez S., FloresRodríguez P., Romero-Ramírez Y., Santos-Bustos N.G. 2019: Factors linked to interannual variation in the metazoan parasite communities of black skipjack, Euthynnus lineatus (Pisces: Scombridae). Invert. Biol. 138: e12259.

Moreno-Sánchez X.G., Quiñonez-Velázquez C., Abitia-CÁrdenas L.A., Rodríguez-Romero J. 2011: Diet of the Pacific sierra Scomberomorus sierra (Perciformes: Scombridae) in two areas of north-west Mexico coast. Int. J. Ichthyol. 17: 185-192.

Oliva M.E., Barrios I., Thatje S., Laudien J. 2008: Changes in prevalence and intensity of infection of Profilicollis altmani (Perry, 1942) cystacanth (Acanthocephala) parasitizing the mole crab Emerita analoga (Stimpson, 1857): an El Niño cascade effect? Helgol. Mar. Res. 62 (Suppl. 1): S57-S62.

Palacios-Fuentes P., Landaeta M.F., González M.T., Plaza G., OJedA F.P., MuÑoz G. 2015: Is ectoparasite burden related to host density? Evidence from nearshore fish larvae off the coast of central Chile. Aquat. Ecol. 49: 91-98.

Pérez-Ponce de León G., García-Prieto L., Mendoza-Garfias B., Leon-Règagnon V., Pulido-Flores G., AranDa-Cruz C., García-Vargas F. 1999: IX. Biodiversidad de helmintos parásitos de peces marinos y estuarinos de la Bahía de Chamela, Jalisco. Listados Faunísticos de México. Universidad Nacional. Autónoma de México, Mexico City, 51 pp.

Poulin R. 2006: Variation in infection parameters among populations within parasite species: Intrinsic properties versus local factors. Int. J. Parasitol. 36: 877-885.

Poulin R., Kamiya T. 2015: Parasites as biological tags of fish stocks: a meta-analysis of their discriminatory power. Parasitology 142: 145-155.

Poulin R., Krasnov B.R., Mouillot D. 2011: Host specificity in phylogenetic and geographic space. Trends Parasitol. 27: 355-361. Poulin R., Rohde K. 1997: Comparing the richness of metazoan communities of marine fishes: controlling for host phylogeny. Oecologia 110: 278-283.

RoHDE K. 2005: Ecology of Marine Parasites. CABI Publishing, Wallingford, $590 \mathrm{pp}$

Rojas-Herrera A.A., Violante-GonzÁlez J., Garcia-Ibáñez S., Villerias-Salinas S., Moreno-Diaz G. 2016: Temporal variation of the pelagic copepod community in Acapulco Bay, México. Open J. Marine Sci 6: 40-48.

Santos-Bustos N.G., Violante-González J., Monks S., Rojas-Herrera A.A., García-Ibáñez S., Flores-Rodríguez P., Almazán-Núñez R.C., Moreno-Díaz G. 2018: Species richness and similarity of metazoan parasite communities in three species of leatherjacket (Oligoplites: Pisces: Carangidae) from the Pacific coast of Mexico. Invert. Biol. 137: 205-220.

Sasal P., Morand S., Guegan J.-F. 1997: Determinants of species of parasite richness in Mediterranean marine fishes. Mar. Ecol. Prog. Ser. 149: 61-71.

Sokal R.R., Rohlf F.J. 1998: Biometry. Second edition. W.H. Freeman and Company, San Francisco, California, 859 pp.

Timi J.T. 2007: Parasites as biological tags for stock discrimination in marine fish from South American Atlantic Waters. J. Helminthol. 81: 107-111.

Timi J.T., Lanfranchi A.L., LuQue J.L. 2010: Similarity in parasite communities of the teleost fish Pinguipes brasilianus in the southwestern Atlantic: infracommunities as a tool to detect geographical patterns. Int. J. Parasitol. 40: 243-254.

Timi J.T., Poulin R. 2003. Parasite community structure within and across host populations of a marine pelagic fish: how repeatable is it? Int. J. Parasitol. 33: 1353-1362.

Timi J.T., Rossin M.A., Alarcos A.J., Braicovich P.E., CanTATORE D.M.P., LANFranchi A.L. 2011: Fish trophic level and 
the similarity of non-specific larval parasite assemblages. Int. J. Parasitol. 41: 309-316.

Villalba-Vásquez P.J., Violante-González J., Monks S., Marino-Romero J.U., García-Ibáñez S., Rojas-Herrera A.A., Flores-Garza R., Rosas-Guerrero V. 2018: Temporal and spatial variations in the metazoan parasite communities of the Panama spadefish, Parapsettus panamensis (Pisces: Ephippidae), from the Pacific coast of Mexico. Invert. Biol. 137: 339-354.

Violante-González J., Gallegos-Navarro Y., Monks S., Garcia-Ibáñez S., Rojas-Herrera A.A., Pulido-Flores G., Villerias-Salinas S., Larumbe-Moran E. 2016. Para- sites of the green jack Caranx caballus (Pisces: Carangidae) in three locations from Pacific coasts of Mexico, and their utility as biological tags. Rev. Mex. Biodiv. 87:1015-1022.

Violante-González J., Monks S., Gallegos-Navarro Y., Santos-Bustos N.G., Villalba-Vásquez P.J., Padilla-Serrato J.G., Griselda Pulido-Flores G. 2020: Interannual variation in the metazoan parasite communities of bigeye trevally Caranx sexfasciatus (Pisces, Carangidae). Parasite 27: 6.

ZANDER C.D. 2004: Four-year monitoring of parasite communities in gobiid fishes of the southwestern Baltic. II. Infracommunity. Parasitol. Res. 93: 17-29.

Cite this article as: Santos-Bustos N.G., Violante-González J., Monks S., Villalba-Vásquez P.J., Villalobos S.S.S, Acosta-Hernández M.S., Gallegos A.D. 2020: Interannual and spatial variation in the parasite communities of Pacific sierra Scomberomorus sierra (Jordan et Starks) on Mexico's Pacific coast. Folia Parasitol. 67: 029. 